\title{
Serial Block-Face Scanning Electron Microscopy and the Reconstruction of Plant Cell Membrane Systems
}

\author{
Maike Kittelmann, Chris Hawes*, Louise Hughes \\ Department of Biological \& Medical Science, Oxford Brookes University \\ Oxford OX3 OBP, UK.
}

*Author for Correspondence

Email: chawes@brookes.ac.uk

Orchid IDs:

CH: 0000-0003-4856-7690

LH: 0000-0002-7505-202X

Key words: SBFSEM, plant membranes, osmium impregnation, selective staining. 


\section{Abstract:}

Serial block face imaging with the scanning electron microscope has been developed as an alternative to serial sectioning and transmission electron microscopy for the ultrastructural analysis of the three dimensional organisation of cells and tissues. An ultramicrotome within the microscope specimen chamber, permits sectioning and imaging to a depth of many microns within resin embedded specimens. The technology has only recently been adopted by plant microscopists and here we describe some specimen preparation procedures suitable for plant tissue, suggested microscope imaging parameters and discuss the software required for image reconstruction and analysis.

\section{Introduction:}

Traditionally cell ultrastructure has been studied by transmission electron microscopy (TEM) with data comprising images obtained from ultrathin resin embedded material, replicas or negatively stained particles. It has, however, long been realised that in reality only a minute volume of prepared material can be imaged by TEM. If three-dimensional or volume information on cell and tissue structure is needed then extremely tedious serial section and $3 D$ reconstruction techniques are required (Bang \& Bang 1957). In an attempt to overcome this problem in the late 60's and 70's high voltage electron microscopes (1-3 MeV) were used to image either extremely thick $1 \mu \mathrm{m}$ plus sections (Glauert 1979, Hawes 1981a,b) or whole freeze- or critically-point dried cells (Porter \& Anderson 1982, Hawes 1985), which were subsequently analysed using stereoscopic viewing and measuring techniques. More recently, electron tomography of semi-thick resin sections has become the technique of choice for high resolution 3D imaging of organelles (Austin \& Staehelin 2011). Whilst it is possible to expand this technique to imaging whole cells using electron tomography combined with serial sectioning (Noske et al., 2008), time and technical limitations preclude the use of this as a routine technique for the $3 D$ analysis of cells and tissues.

In the last decade three new technologies for TEM level resolution biological imaging have been developed, based around a combination of high resolution field emission scanning electron microscopy (FEGSEM) and the backscattered electron (BSE) imaging of the surface of sections or blocks of resin-embedded material. The use of BSE to image stained and resin embedded samples allows larger areas of tissue to be captured in a single image or for image montaging (Rizzo et al., 2015) and creates TEM-like images. Array tomography extends this BSE imaging modality to serial sections. Unlike TEM, the sections used for 
array tomography are not restricted to grids but can be collected onto tape using a system such as the automatic tape-collecting ultramicrotome (ATUM) or other substrates such as glass slides (Wacker et al., 2015). Used in combination with automatic imaging techniques, data from many thousands of sections can be collected, spanning large volumes of tissue. Array tomography offers the potential to archive material, add additional stain to sections if required and provide a mechanism for correlative microscopy. The technique relies on the physical collection of sections and can have artefacts commonly associated with producing sections, for example, compression, alignment issues, missing sections and stain precipitation, but has yet to be applied to plant material.

Focussed ion beam (FIBSEM) uses a gallium ion beam to physically etch (mill) away resin from the surface of the specimen block. The accuracy of this technique permits effective "sectioning" of the block in steps as fine as $5 \mathrm{~nm}$ (Kremer et al., 2015) and has been used to successfully reconstruct Golgi stacks in unicellular algae (Wanner et al., 2013). Thus, high resolution backscattered electron data can be collected from what are effectively very thin sections, sub $5 \mathrm{~nm}$, making this technique almost comparable with electron tomography in a TEM, but with the advantage that there is, other than time constraints, little limitation to the depth at which the resin block can be milled. The disadvantage of this technique is the limited area that can be imaged in one region of interest $\left(\sim 50 \mu \mathrm{m}^{2}\right)$. Additionally, there is a significant amount of expertise required to run the system as well as cost of the instrumentation and subsequent maintenance.

Another approach, which has recently seen a rise in popularity (Kremer et al., 2015, Borrett \& Hughes, 2016) is based around serial block face scanning electron microscopy (SBFSEM). Here, an ultramicrotome with a diamond knife is installed in the chamber of an SEM and sections as thin as $15-20 \mathrm{~nm}$ can be removed from the block face and discarded prior to block face imaging. The instrumentation for this, manufactured by Gatan (Abingdon, UK), is marketed as the 3-View (Denk \& Horstmann 2004) and can be retrofitted to appropriate scanning electron microscopes. In comparison to FIBSEM, removal of sections by ultramicrotome is faster than milling, images from larger regions of interest (only restricted by the degree of stage movement, $600 \times 600 \times 600 \mu \mathrm{m})$ can be collected and the instrumentation is easier to use. However, with current systems the achievable resolution in $z$ (limited by slice thickness and the depth of signal collection from within the block) is not as high as with FIBSEM. Both FIBSEM and SBFSEM suffer from the disadvantage of being a destructive technique. Where in FIBSEM regions that have been imaged including the trench cannot be revisited, the entire block-face is discarded in SBFSEM. 
With all of these approaches data collection is fully automated and the instrumentation can be left running for the duration of the experiment. Software is available to permit autocorrelation between the images for alignment and for 3D reconstruction of datasets.

Only recently, has SBFSEM been applied for the three-dimensional imaging of plant tissue (Pollier et al., 2013, Dettmer et al., 2014; Fendrych et al., 2014; Furuta et al., 2014; Scheuring et al., 2016), but combined with selective contrasting techniques, there is great potential for investigating the spatial organisation and interactions of cells and organelles in plants (Hughes et al., 2014). SBFSEM provides the advantage of imaging relatively large areas and volumes, which is especially advantageous, for instance, when imaging large leaf cells. Here we review some of the specimen preparation techniques that can be applied for block face scanning electron microscopy of plants, along with the protocols for using the instrumentation and reconstructing the inevitably large datasets.

\section{Methods:}

All osmium impregnated samples shown in the figures used to illustrate in this review were fixed and prepared according to Materials and Methods outlined in Scheuring et al., (2015) and Hawes et al., (1981b). Most of the SBFSEM data presented here were collected with a ZEISS Merlin Compact VP SEM (Carl Zeiss Ltd, Cambridge, UK), fitted with a Gatan 3View 2XP system. The imaging conditions used were as follows. Maize root (Fig $3 C$ and D) was imaged with an accelerating voltage of $4 \mathrm{kV}$, a variable pressure of $45 \mathrm{~Pa}$, aperture size of $30 \mu \mathrm{m}$, a pixel size of $4.2 \times 4.2 \mathrm{~nm}$, sectioned with a slice thickness of $100 \mathrm{~nm}$. The horizontal field width (HFW) was $16.8 \mu \mathrm{m}$, and the beam dwell time $3 \mu \mathrm{s}$. The imaging conditions for the Arabidopsis root (Fig. $5 \mathrm{~A}$ and B) were $4 \mathrm{kV}, 55 \mathrm{~Pa}$, aperture size $30 \mu \mathrm{m}$, pixel size $6.2 \times 6.2$, slice thickness $50 \mathrm{~nm}$, HFW $24.8 \mu \mathrm{m}$ and dwell time $3 \mu \mathrm{s}$. Similar imaging conditions were used for the pea root (Fig. 7) except with a pixel size of $10.5 \times 10.5$ $\mathrm{nm}$, slice thickness $75 \mathrm{~nm}$ and HFW $9 \mu \mathrm{m}$. The bean leaf (Fig. $5 \mathrm{C}$ and D) was imaged using a variable pressure setting of $40 \mathrm{~Pa}$, a pixel size of $3.3 \times 3.3 \mathrm{~nm}$, a slice thickness of $70 \mathrm{~nm}$ and HFW of $9.9 \mu \mathrm{m}$. Data was stacked, aligned, segmented and processed as described in the text here and as in Scheuring et al., 2015 and Borrett and Hughes (2016). A complete workflow for use of the SBFSEM from fixation to data analysis is given in figure 1.

\section{Specimen Preparation:}


A limiting factor in preparing tissue for SBFSEM is that all contrasting of the specimen must be carried out prior to block imaging and the backscattered signal is often of low contrast, which can only partially be overcome by carefully choosing the correct imaging settings (see below). Plant tissue is especially difficult in this respect as on occasions cell walls and cuticles can obstruct diffusion of fixative and stain into the cells. This means that conventional aldehyde fixation followed by secondary fixation in osmium tetroxide, even if followed by en bloc staining with uranyl acetate, may not be satisfactory in terms of contrast and post-embedding staining is not an option. Thus, some form of extra contrast enhancement is required (Fig. 1). The animal microscopy community has experimented with a range of different fixation protocols which can include extended osmication, reduction of osmium with ferrocyanide or thiocarbohydrazide, and combinations of en bloc staining with uranyl and lead salts (Deerinck et al., Kremer et al., 2015). Unfortunately very few of these different protocols have been either used or their usefulness assessed in plant tissues to date (Kremer et al., 2015).

Different resins are commonly used to embed specimen for SBFSEM. For plants Spurr's is the resin of choice due to its low viscosity that allows better infiltration through the cell walls (Fendrych et al., 2014, Scheuring et al., 2016). It additionally has the advantage of being a very hard resin and such tends to withstand the electron beam better than other resins. However Durcupan has also been successfully used with plant specimen (Pollier et al., 2013, Dettmer et al., 2014, Furuta et al., 2014).

\section{Selective Staining techniques for the endomembrane system.}

For the 3D study of the plant endomembrane system various osmium impregnation techniques have been used in the past, in combination with thick sectioning and high voltage electron microscopy (Harris 1979, Hawes 1981 b). They rely on the reduction of osmium tetroxide on the surface of membranes or in cisternal spaces. The reduction of osmium tetroxide is chemically aided by potassium ferrocyanide (Hepler 1980) or zinc iodide (ZIO) (Hawes 1981a, b, Hawes 1991). The ZIO technique is suitable for the selective staining of higher plant, algal and fungal tissues and has been combined with thick sectioning in a number of studies on the endomembrane system (e.g. Harris 1979, Hawes 1981 a,b). This technique can be used for the impregnation of nuclear envelopes, ER (Fig. 2, 3 c,d), Golgi bodies (Fig. 8), tonoplasts (in some cells), plastid thylakoids (Fig. 5b) and sometimes the stroma of mitochondria. Incubation times depend heavily on the plant specimen and tissue and need to be optimized accordingly to avoid precipitation and overstaining. Microwave- 
assisted sample preparation can increase diffusion efficiency and time and thus optimize ultrastructural preservation and processing time of plant material (Heumann, 1992, Zechmann and Zellnig, 2009).

Osmium impregnated material is perfect for SBFSEM imaging as it already presents a very conductive specimen, thus reducing block face charging. Images with selective contrast are also ideal for rapid segmentation and reconstruction (see below). Images can relatively easily be obtained at a similar quality to conventional thin section electron microscopy at both low magnifications (Fig. 2 c) and intermediate magnification (Fig. 2 a,b). The major advantage over imaging thick sections with high or intermediate voltage TEM is that the limitation on $Z$ thickness from the SBFSEM is only governed by the total $Z$ movement of the stage, which is around $600 \mu \mathrm{m}$ and can be reset to continue data collection for the full depth of the sample. Such a dataset is not realistic with many tissues but it is easily possible to mine a dataset of 20-40 $\mu \mathrm{m}$. A comparison between thick section images of ER and SBFSEM images is given in Figure 3.

\section{Other techniques.}

A whole host of other selective contrasting techniques are available most of which have yet to be applied to SBFSEM studies in plants. The double osmium technique (OTO) combined with thiocarbohydrazide developed for mammalian tissues in the Ellisman lab (protocol available online, Deerink et al. 2010) has been used for the study of sieve plate development (Dettmer et al., 2014). However, the field of enzyme cytochemistry which involves the preembedding contrasting of sites of enzymic activity is eminently applicable to SBFSEM. It is relatively straightforward to locate sites of activity of enzymes such as ATPases or phosphatases to membranes or organelles by precipitating osmium or lead salts after reaction with an appropriate enzyme substrate (see Sexton and Hall 1991 for numerous examples). Likewise cell walls can be contrasted by the periodic acid-thiocarbohydrazidesilver proteinate (PATAg) technique (Roland and Vian 1991). Many of these and similar techniques have been around for many years and although rarely used nowadays could prove useful for semi-automated reconstruction of plant cell datasets. For instance, in the past we have used a uranyl acetate, copper and lead citrate impregnation technique to selectively contrast membranes and polysaccharides (Hawes \& Horne 1983), and this can be used for reconstructing membrane-bounded organelles (Fig. 4).

\section{Use of the microscope system:}




\section{Mounting and trimming the resin block.}

After selecting the fixation, staining and embedding techniques, as outlined above, the sample is mounted onto the flat surface of a specimen pin, carefully trimmed to appropriate size prior to inserting it into the microscope stage (Fig. 1). The specimen pin or "rivet" is available in two designs, flat and pointed (Fig. 6), to accommodate samples up to $2 \mathrm{~mm}$ in height. The resin block should be trimmed, using a fine jeweller's saw, so that it is roughly the same dimension as the platform on the specimen pin (Fig. 6). Orientation of the sample at this stage is crucial as the specimen pin cannot be tilted in the microtome, and the sample must be trimmed and mounted with the desired orientation in mind. A conductive adhesive resin (e.g. Circuitworks conductive epoxy - catalogue number CW2400, ITW Chemtronics, Georgia, USA) can be used to attach the sample to the pin. Once mounted the resin and glue needs to be fully cured before the block is trimmed, either by leaving the pins overnight or curing the epoxy resin for an hour at $70^{\circ} \mathrm{C}$ and cooling to room temperature. The block face can then either be shaped using the edge of a trimming knife in an ultramicrotome or by using a new razor blade. The block face needs to be less than $1 \mathrm{~mm}$ in diameter and ideally should be as small as possible, keeping the amount of free resin around the sample to a minimum. Conductive Epoxy glue should be removed from any areas that will be sectioned in the SBFSEM as it tends to stick to the diamond knife. The height of the resin block must be small enough to fit between the lowest setting on the stage and the diamond knife. Our blocks are normally mounted onto the flat pins and are typically $1.5-2 \mathrm{~mm}$ in height from the specimen pin (Fig. 6). If the sample allows, the final stage in trimming the sample is to smooth the block face with a glass or diamond knife parallel to the face of the pin. This will facilitate an easier alignment of the sample to the diamond knife in the 3 view stage.

Variable pressure systems do an excellent job of eliminating charge from the surface of a resin block. Provided the sample is stained with heavy metal and has only minimal regions of empty resin, coating the sample may not be necessary. However, in samples where there are large areas of resin, such as plant tissue with large vacuoles, coating helps to dissipate charge build up. This allows imaging of the sample under higher vacuum, thus improving the resolution that can be achieved. We typically use $15-20 \mathrm{~nm}$ of gold coating on our samples, sputter coated as in normal SEM sample preparation. Platinum coating can also be used (Fig. 1, Pollier et al., 2013, Dettmer et al., 2014; Fendrych et al., 2014; Furuta et al., 2014). The block face being imaged is cut with the diamond knife and the gold coating does not interfere with signal from the stained sample. In SEM, silver dag or paint is often used around the edge of the specimen stub to improve conductivity. Silver dag can soften the resin and in our experience this makes the sample block more prone to cutting artefacts once in the microscope and thus we do not advise its use for SBFSEM samples. Ideally the 
cutting window of the knife should have some leeway before and after the block face, which can be achieved by adjusting the knife position. Mounting the specimen pin in the stage and approaching the knife is a relatively simple procedure conducted while the SEM chamber door is open. Similar principals to that employed in ultramicrotomy are used in setting the cutting window and slowly approaching the knife edge.

The final approach is conducted using the automated system in the 3view software. Once most of the resin block surface is being cut the SEM door can be closed and imaging can begin.

\section{Imaging the block face.}

Imaging parameters are going to vary depending on the type of instrumentation available and system set up. Most of the SBFSEM data presented here were collected with a ZEISS Merlin Compact VP SEM (Carl Zeiss Ltd, Cambridge, UK), fitted with a Gatan 3View 2XP system (Figs. 2C 3C, 5 and 6). Specific imaging conditions for the figures are included in the methods section above. Key microscope parameters are accelerating voltage, variable pressure, magnification, focus and aperture size. Beam dwell time, specimen position, pixel size, horizontal field width (field of view), slice thickness, brightness and contrast are controlled through the 3view system (Fig. 1). The voxel size of the final data is dependent on the set pixel size and slice thickness determining the theoretical $z$ resolution. There is a physical limitation on how thin of a slice a diamond knife can cut (we have cut as thin as 35 $\mathrm{nm}$ ). However, most biological questions addressed by SBFSEM will be satisfied using slices thicknesses between 40 and $100 \mathrm{~nm}$ for plant tissue (Furuta et al., 2014; Scheuring et al., 2016), thus voxels tend to have a higher pixel resolution in $x$ and $y$ than in $z$ (Peddie and Collinson, 2014),

The final resolution in the data depends on how well the sample has been contrasted and other imaging parameters used. Changing the accelerating voltage of the beam has a significant effect of the depth of beam penetration into the sample and the amount of detail that can be resolved as a result (Knott et al., 2008). Lower accelerating voltages are considered better for resolution, but also lower the amount of signal to noise in the image (Fig. 7). A balance needs to be achieved, especially in samples that have lower contrast. The beam dwell time, measured in $\mu \mathrm{s}$, also has an impact on the resolution, with a higher dwell time producing a better signal to noise ratio than a lower one (Denk and Horstmann, 2004). Beam penetration and dwell time can also have an effect on the integrity of the specimen being imaged. Resin sections show loss of mass and shrinkage when exposed to an electron beam in the TEM (Stenn and Barr, 1970). Similarly radiation damage occurs when imaging the block face and can interfere with the sectioning properties of the sample, 
causing uneven sections, skipped sections or even progressive and visible damage to the surface. Lowering the beam voltage, reducing the aperture size and reducing beam dwell time can mitigate radiation damage and improve sectioning qualities of the sample, offset by the disadvantage of a drop in the signal to noise ratio (Fig. 7, Starborg et al., 2013).Specimens with large areas of empty resin, such as vacuolated cells, seem to be more sensitive to radiation damage. Changing the type of resin used to embed the sample or increasing the hardness of the resin can improve resistance to beam induced damage (Kizilyaprak et al., 2015). We routinely use Spurr's hard mix (TAAB, Berkshire, UK) for plant samples. Microscope parameters that have successfully been used with plant tissue are accelerating voltages of 2.5 to $4 \mathrm{kV}$ and a pixel dwell time of 3 to $8 \mu \mathrm{s}$, and a variable pressure of 20 to $50 \mathrm{~Pa}$ ( 0.15 to 3.8 Torr), depending on the magnification used and the tissue type being imaged (Dettmer et al, 2014; Furuta et al., 2014; Scheuring et al., 2016, see also Figs. 2-5). Variable pressure to reduce charging is the method of choice for plant material, especially tissues that contain large amounts of empty resin. However, lower kV (1.5) and high vacuum is possible for some plant specimens (Fendrych et al., 2014), especially for lower magnification datasets. The standard aperture size for the 3 View system is $30 \mu \mathrm{m}$, but we routinely use the $20 \mu \mathrm{m}$ aperture when imaging plant tissue with selective endomembrane staining (see above and Figs. 2-5). Figure 7 shows images of the same block face taken at a range of $\mathrm{kVs}$ and pressures with two different final column aperture sizes demonstrating changes in contrast under different imaging conditions.

\section{Data handling and storage:}

As SBFSEM is a destructive technique and the localisation of a specific structure or event cannot always be determined before image collection, oversampling is a common approach. Larger areas of the block face can be imaged at lower magnifications using high resolution images (for example, $10,000 \times 10,000$ pixels with a dwell time of up to $8 \mu \mathrm{s}$ ). Sub-areas of the dataset can later be extracted, still providing enough resolution to perform image analysis or segmentation. Thus, single images of the original dataset can easily reach 100 MB or more. Alternatively concurrent data-sets can be collected by imaging multiple regions of interest on the block face. The problems arising are obvious. Imaging times can be long and the computers for data collection as well as those used for image processing need to have sufficient memory and a suitable graphics board to handle large datasets.

Consequently, data storage and data management is becoming a pressing issue. Considering the number of GBs potentially generated on a daily basis, the data storage 
problem needs to be addressed at the user, facility and Institutional level and should be taken into consideration when planning research with or purchasing of an SBFSEM. There are open source or commercial databases available that allow storage and also remote access, sharing, management and even analysis of data, for example, iPlant (Goff et al., 2011). Other options are reviewed in Eliceiri et al., 2012. If SBFSEM data is to be processed locally, the computers (in our experience) should have a minimum of 16 GB RAM, multiple CPU, ideally an SSD drive and a graphics board with a minimum 2GB RAM to allow reasonable processing times and image-processing software to run properly. It is good practise to check the computing requirements for the software that will be used to process data as this can be extremely memory intensive. With the explosion of 3D data collected the electron microscopy community as well as the bioimaging community in general is discussing how raw data could or should be shared for further mining and with initiatives such as the Cell Centered Database (CCDB) and the Open Microscopy Environment this has become a reality (Martone et al., 2008, Allan et al. 2012). However, issues of storage possibilities, costs, management, technical installation and maintenance, annotation, ownership and credit are not easily solved for a world-wide community.

\section{Data processing and analysis}

After a dataset is obtained it usually has to go through several pre-processing steps before features of interest can be extracted and analysed (Fig. 1). These steps often involve the transformation of the file format from a system or software specific format to a more widely compatible file format or a stack format. We usually record the data using Digital Micrograph (Gatan, Oxfordshire, UK) format (.dm4) and then convert image files into mrc-stacks using the dm2mrc command in etomo (IMOD, Boulder, Colorado, USA; Kremer et al., 1996; Table 1). Information for $z$ then needs to be corrected to the slice thickness using the alterheader command. Series of 2D images can be used for further processing, however stacks may be easier to handle and process for some programs, for example, when using the data processing functions of etomo (Kremer et al., 1996). For an initial inspection of the dataset we bin the stack up to 8 times, determine the sub-volumes of interest and extract them later from original datasets using the trimvol command in etomo.

Processing sub-volumes typically starts with section alignment (manual or automatic). Although the images collected from SBFSEM are generally well aligned due to the nature of the technique, slight shifts can often be observed. Failure to correct for these shifts leads to the introduction of artefacts and skewed 3D models, especially when segmenting small and detailed objects such as Golgi bodies or the endoplasmic reticulum (Thierry et al., 2014). A 
number of programs are available to perform the alignment; the most commonly used are listed in Table 1. We use automatic alignment algorithms using a Hanning window filter in Digital Micrograph or the etomo graphics user interface alignment of serial sections (Kremer et al., 1996) with default parameters and only rotation and translation. Subsequently, application of filters (median filter in etomo, Gaussian filter etc.) and adjustment of contrast and brightness can be useful to reduce noise and enhance the signal. These can be applied in the programs used for image pre-processing after alignment and stacking or in segmentation programs, where the output is immediately visible next to the original data (Table 1).

Segmentation can be done in most image processing programs (see Table 1), but can be slow, subjective and a significant bottle neck in data processing (Peddie and Collinson, 2014). The time and labour involved in manually tracing even just one feature over multiple slices/images is often not cost effective, although maybe necessary to address some research questions, especially if selective contrasting of sub-cellular structures cannot not been undertaken.

There are a number of (semi-) automated approaches available in different image processing programs (see Table 1 ) to make the segmentation process easier, quicker and more objective. Masking followed by pixel-based thresholding (determination of a region of interest within which auto-segmentation can take place) as available in Microscopy Image Browser (Belevich et. al. 2016), Amira (FEI, Cambridge, UK), Imaris (Bitplane AG, Zurich, Switzerland) (see figure 8) is very effective when combined with the selective staining techniques outlined above and can be conducted in a matter of minutes. Region growth (magic wand or wildfire tools found in Microscopy Image Browser, Amira, Imaris and Reconstruct (Fiala, 2005), amongst others) only thresholds connected pixels of a specified range from a seed point and thus allows more manual interference. Pixel and object classification (e.g. used in llastik, Sommer et al., 2011) makes the process quicker and less subjective, especially when a larger number of similar datasets need to be segmented. However, a certain amount of time for the initial machine learning has to be spent which involves manual corrections of the suggested segmentation. Edge detection is a filter available in most image processing programs to detecting boundaries of objects and can be used to enhance the signal to noise ratio before further automated segmentation. However, because all features in an EM dataset consist of grey pixels, it can be difficult to detect clear boundaries between features of similar grey value. Watershed is one technique that can be used to distinguish separate features more objectively (available for example in llastik). An EM image is viewed as a topographic relief, pixels with low intensity representing low and high intensity representing high landmarks. The "landscape" is slowly filled with "water". 
Where two water bodies meet, a line is put in place, thus marking the division between two structures. This technique is normally used on 2D images (Hagyard et al., 1996). However, Volkmann (2002) developed a new variant applicable to 3D EM data where the operation starts at the global density minimum and expands in a voxel-by-voxel based manner, checking for connectivity to other water bodies at each step. Carving as used in llastik is a seeded watershed algorithm where only a selected object is segmented in 3D using watershed.

Specific algorithms are often developed according to specific scientific questions to detect a structure of interest. Tracing of tubular structures or filaments is probably the most common option available even in commercial programs and mostly useful for automatic microtubule but also neurite detection (Frangi tubular filter in Microscopy Image Browser, XTracing in Amira).

Due to the broad range of samples and structures that need to be segmented, a combination of techniques is commonly used. It should be noted that all image-processing tasks alter the original data and thus potentially introduce artefacts.

Once segmented, it is often desired to render 3D models from the selected features for quantifications such as volume or surface area, number and distances to other structures, but also for simple visualization. Depending on the software there are usually a variety of options to edit the models in terms of colour, transparency and smoothness or even create a stereo-view or animation including images from the original data (see Table 1).

Plant research utilising SBFSEM has used the open source programs IMOD, ImageJ/FIJI, Microscopy Image Browser (Belevich et. al. 2016) and Ilastik (Sommer et al., 2011) and the commercially available Amira (Pollier et al., 2013, Dettmer et al., 2014; Fendrych et al., 2014; Furuta et al., 2014; Scheuring et al., 2016). With the selective staining of the endomembrane system using the $\mathrm{ZIO}$ protocol we are often able to use a maximum intensity projection based on thresholding as a very quick method to visualize the ER using Amira or Imaris. However, a wide variety of other programs are available and these have been employed to process SBFSEM data in different tissues and organisms as reviewed by Borrett and Hughes (2016). There are advantages and disadvantages to both Open Source and Commercial software packages. Open Source software is free and general packages such as etomo (IMOD), ImageJ/Fiji or Microscopy Image Browser can be endlessly extended in functionality through macros and plugins to deal with the specific questions and demands of the individual researcher. The Microscopy Image Browser provides a broad range of functions for image pre-processing as well as manual and automated segmentation and can use plugin extensions. With a more and more user-friendly interface llastik is 
becoming especially powerful in terms of segmentation of large datasets with large numbers of features that need to be extracted by using machine-based pixel and object classification. In most open access programs bugs tend to be fixed more quickly and a large active community is often available to help with questions and problems. However, the sheer quantity of plugins and updates can make it difficult, especially for beginners, to find a good way of utilising and customizing the program. Additionally the ability to write code or use of command lines may be required. Although most programs will be more suitable for either image processing or segmentation or visualization, open source programs often allow the transfer of files from one program to another or are built on top of each other and thus complement each other to go from pre-processing to 3D model generation.

Amira ( $\mathrm{FEl}$, Cambridge, UK) is probably one of the most commonly used commercial programs for EM data analysis and visualisation. After data collection on the microscope it can do all following steps up to 3D model generation including stereo-views and animations (Fig. 8). Extensions can be bought that yield additional image analysis functions. The most relevant extensions for SBFSEM data are probably XVolume to allow processing of large datasets that exceed the memory available in the hardware, Xtracing to detect and trace filaments or tube-like structures and XPand to develop own extensions in C++ (Amira FEl, Cambridge, UK). The compact availability of a variety of functions and a user-friendly interface do however come with a significant cost.

Imaris is another modular software package commercially available from Bitplane (Bitplane AG, Zurich, Switzerland, Fig. 8). However, it is primarily designed for fluorescence microscopy data and much more powerful in terms of segmentation and visualization on this type of dataset. However, given a selective stain of the structure of interest, semi-automated threshold rendering is straightforward and user-friendly in Imaris (e.g. Bohorquez et al., 2014; Shigetomi et al., 2013; Zhuravleva et al., 2012; Rouquette et al., 2009).

Both Amira and Imaris software packages have ability to rapidly produce quantitative date from segmented images such as organelle volume and membrane surface area. The programs most commonly used for SBFSEM data processing and visualisation are listed in table 1, including information about their abilities and limitations. We hope that this overview is helpful especially for new users in the SBFSEM field who need to decide on which software program to use for the different tasks.

\section{The future:}


It is certainly early days in the application of SBFSEM and indeed FIBSEM technology to plant specimens (see Lutz-Meindle et al., this edition). Here we have described the application of two selective staining techniques to generate sufficient contrast in plant specimens to permit rapid (semi-) automatic segmentation, using proprietary software, of numerous serial images through cells and tissues. However, many other approaches can

potentially be made to selectively highlight individual organelles in the cytoplasm. For instance, the whole gamut of enzyme cytochemistry techniques, popular in the nineteen seventies and eighties, could be used (see Sexton and Hall 1991) to selectively highlight areas of enzyme activity in the cell. Immunocytochemistry using osmium reduction instead of gold labelling is a possibility, as is the photooxidation of DAB by fluorescent proteins (Meisslitzer et al., 2008) or other fluorescent tags such as miniSOG (Shu et al., 2011) or the use of genetically encoded probes with enzyme rather than fluorescent protein tags (Martell et al., 2012). It should however be noted that the sheer abundance of plant endogenous peroxidases may limit the use and specificity of such genetically encoded enzyme tags in plants.

As specimen charging can be a problem with vacuolated tissues, the development of conductive resins or methods that increase the conductivity of traditionally used resins (Nguyen et al., 2016) may permit the use of lower kVs and better vacuums and thus enable higher resolution datasets from such samples, which will be especially beneficial for plant research.

More recently the technique of array tomography has been introduced, whereby high resolution field emission scanning electron microscopy can be used to image serial sections for subsequent reconstruction (Micheva \& Smith 2007, Wacker et al., 2015). To date, this has been the preserve of the neuroscientists and is yet to be exploited by the plant community, but has great potential for combining immunogold labelling with 3D reconstruction.

Perhaps the ultimate aim will be to combine these staining and labelling techniques with ultra-rapid cryo-fixation of specimens, which has yet to be achieved. Certainly this combination of technologies both old and new will herald a new era in our understanding of the ultrastructural organisation of plant cells.

\section{Acknowledgements:}

We thank the BBSRC for an ALERT13 award to $\mathrm{CH}$ for purchase of the Zeiss Merlin Compact SEM with Gatan 3View system (BB/L014122/1). Some of the work was also 
supported by an ERACAPs/BBSRC grant BB/M000168/1 to $\mathrm{CH}$. We acknowledge Gatan and Sandy Monteith for organising data collection for use in Figure 3.

\section{References:}

Allan, C., Burel, J.M,, Moore, J., Blackburn, C., Linkert, M., Loynton, S., Macdonald, D., Moore, W.J., Neves, C., Patterson, A., Porter, M., Tarkowska, A., Loranger, B., Avondo, J., Lagerstedt, I., Lianas, L., Leo, S., Hands, K., Hay, R.T., Patwardhan, A., Best, C., Kleywegt, G.J,, Zanetti, G. \& Swedlow, J.R. (2012) OMERO: flexible, model-driven data management for experimental biology. Nat Methods. 9, 245-53.

Austin, J.R. \& Staehelin, L.A. (2011) Three-dimensional architecture of grana and stroma thylakoids of higher plants as determined by electron tomography. Plant Physiol. 155, 1601-11.

Bang, B., H. \& Bang, F.B. (1957) Graphic reconstruction of the third dimension from serial electron microphotographs. J Ultrastruct Res. 1, 138-139

I. Belevich, M., Joensuu, D., Kumar, H., Vihinen and E. Jokitalo. (2016) Microscopy Image Browser: A platform for segmentation and analysis of multidimensional datasets. PLoS Biology 14, e1002340.

Bohorquez, D. V., Samsa, L. A., Roholt, A., Medicetty, S., Chandra, R. \& Liddle, R. A. (2014) An enteroendocrine cell-enteric glia connection revealed by $3 \mathrm{D}$ electron microscopy. PLoS On, 9, e89881.

Borrett, S. \& Hughes, L. (2016) Reporting methods for processing and analysis of data from serial block face scanning electron microscopy. J. Microsc., doi: 10.1111/jmi.12377.

Deerinck, T.J., Bushong, E.A., Thor, A. \& Ellisman, M. H. NCMIR methods for 3d EM: a new protocol for preparation of biological specimens for serial block face scanning electron 
microscopy. http://www.dsimagingllc.com/wp-content/uploads/2013/04/OTO-fixationfor-SEM-Imaging-MW.pdf

Denk, W. \& Horstmann, H. (2004) Serial block-face scanning electron microscopy to reconstruct three-dimensional tissue nanostructure. PLoS Biol, 2, e329.

Dettmer, J., Ursache, R., Campilho, A., Miyashima, S., Belevich, I., O'Regan, S., Mullendore, D. L., Yadav, S. R., Lanz, C., Beverina, L., Papagni, A., Schneeberger, K., Weigel, D., Stierhof, Y. D., Moritz, T., Knoblauch, M., Jokitalo, E. \& Helariutta, Y. (2014) CHOLINE TRANSPORTER-LIKE1 is required for sieve plate development to mediate long-distance cell-to-cell communication. Nature Comm. 5, 4276.

Eliceiri, K.W., Berthold, M.R., Goldberg, I.G., Ibáñez, L., Manjunath, B.S., Martone, M.E., Murphy, R.F., Peng, H., Plant, A.L., Roysam, B. \& Stuurman, N., 2012. Biological imaging software tools. Nature Methods 9, 697-710.

Fendrych, M., Van Hautegem, T., Van Durme, M., Olvera-Carrillo, Y., Huysmans, M., Karimi, M., Lippens, S., Guérin, C.J., Krebs, M., Schumacher, K. \& Nowack, M.K. (2014) Programmed cell death controlled by ANAC033/SOMBRERO determines root cap organ size in Arabidopsis. Current Biol, 24, 931-40.

Fiala, J.C., 2005. Reconstruct: a free editor for serial section microscopy. J. Microscopy, 218, 52-61.

Furuta, K. M., Yadav, S. R., Lehesranta, S., Belevich, I., Miyashima, S., Heo, J. O., Vaten, A., Lindgren, O., De Rybel, B., Van Isterdael, G., Somervuo, P., Lichtenberger, R., Rocha, R., Thitamadee, S., Tahtiharju, S., Auvinen, P., Beeckman, T., Jokitalo, E. \& Helariutta, Y. (2014) Plant development. Arabidopsis NAC45/86 direct sieve element morphogenesis culminating in enucleation. Science, 345, 933-937.

Goff, S.A., Vaughn, M., McKay, S., Lyons, E., Stapleton, A.E., Gessler, D., Matasci, N., Wang, L., Hanlon, M., Lenards, A. Muir, A. et al. (2011) The iPlant collaborative: cyberinfrastructure for plant biology. Frontiers Plant Sci. 2, 34 . doi: 10.3389/fpls.2011.00034.

Glauert, A.M. (1979) Recent advances of high voltage electron microscopy in biology. J. Microsc. 117, 93-101. 
Hagyard. D', Razaz, M., \& Atkin, P. (1996) Analysis of watershed algorithms for greyscale images. Proc. IEEE Intl. Conf. Image Process. 3, 41-44

Harris, N. (1979) Endoplasmic reticulum in developing seeds of Vicia faba. A high voltage electron microscope study. Planta 146, 63-69.

Hawes. C., R. (1981a) High voltage electron microscopy of biological materials. Micron 12, 159-160.

Hawes, C.R., Juniper, B. \& Horn, J,C. (1981b) Low and high voltage electron microscopy of mitosis and cytokinesis in maize. Planta 152, 397-407.

Hawes. C. R. \& Horne, J. C. (1983) Staining plant cells for thick sectioning: uranyl acetate, copper and lead citrate impregnation. Biol Cell 48, 207-210.

Hawes, C.R. (1985) Conventional and high voltage electron microscopy of the cytoskeleton and cytoplasmic matrix of carrot (Daucus carota L.) cells grown in suspension culture. Europ. J. Cell Biol. 38, 201-210.

Hawes, C. (1991) Stereo-electron microscopy. Chp. 2 in Electron Microscopy of Plant Cells (Eds. C Hawes \& J I Hall) pp 67-84. Academic Press, London.

Hepler, P., K. (1980) Membranes of the mitotic apparatus of barley cells. J Cell Biol 86, 490499.

Heumann, H.-G. (1992) Microwave-stimulated glutaraldehyde and osmium tetroxide fixation of plant tissue: ultrastructural preservation in seconds. Histochemistry 97, 341-347.

Hughes, L., Hawes, C., Montieth, S \& Vaughan, S. (2014) Serial block face scanning electron microscopy - the future of cell ultrastructure imaging. Protoplasma 25, 395-401.

Kizilyaprak, C., Longo, G., Daraspe, J. \& Humbel, B.M. (2015) Investigation of resins suitable for the preparation of biological sample for 3-D electron microscopy. J. Struct. Biol. 189, 135-146.

Knott, G., Marchman, H., Wall, D. \& Lich, B. 2008. Serial section scanning electron 
microscopy of adult brain tissue using focused ion beam milling. J. Neurosci. 28, 29592964.

Kremer, A., Lippens, S., Bartunkova, A., Asselberghs, B., Blankpain, C., Fendrych, M., Goosens, A., Holt, M., Janssens, S., Krols, M., Larsimont, J. -C., McGuire, C., Nowack, M.K., Saelens, X., Schertel, A., Schepens, B., Slezak, M., Timmermans, V., Theunis, C., van Brempt, R., Visser, Y. \& Guérin, C. (2015) Developing 3D SEM in a broad biological context. J. Microsc. 259, 80-96.

Kremer, J.R., Mastronarde, D.N. \& McIntosh, J.R. (1996) Computer visualization of threedimensional image data using IMOD. 116, 71-76.

Martell, J.D., Deerinck, T.J., Sancak, Y., Poulos, T.L., Mootha, V.K,, Sosinsky, G.E., Ellisman, M.H. \& Ting AY.(2012) Engineered ascorbate peroxidase as a genetically encoded reporter for electron microscopy. Nat. Biotechnol. 30,1143-1148.

Martone, M.E., Tran, J., Wong, W.W., Sargis, J., Fong, L., Larson, S., Lamont, S.P., Gupta, A., Ellisman, M.H. (2008) The cell centered database project: an update on building community resources for managing and sharing 3D imaging data. J Struct Biol. 3, 220231

Meisslitzer-Ruppitsch, C., Vetterlein, M., Stangl, H., Maier, S., Neumüller, J., Freissmuth, M., Pavelka, M. \& Ellinger A. (2008). Electron microscopic visualization of fluorescent signals in cellular compartments and organelles by means of DAB-photoconversion. Histochem Cell Biol. 130, 407-419.

Micheva, K.D. \& Smith, S.J. (2007). Array tomography: a new tool for imaging the molecular architecture and ultrastructure of neural circuits. Neuron 55, 25-36.

Nguyen, H.B., Thai, T.Q., Saitoh, S., Wu, B., Saitoh, Y., Shimo, S., Fujitani, H., Otobe, H. and Ohno, N., (2016). Conductive resins improve charging and resolution of acquired images in electron microscopic volume imaging. Scientific Reports, 6, 23721.

Noske, A.B., Costin, A.J., Morgan, G.P. and Marsh, B.J., (2008). Expedited approaches to whole cell electron tomography and organelle mark-up in situ in high-pressure frozen pancreatic islets. J. Structural Biol. 161, 298-313. 
Peddie, C.J. and Collinson, L.M. (2014) Exploring the third dimension: volume electron microscopy comes of age. Micron, 61, 9-19.

Pollier, J., Moses, T., González-Guzmán, M., De Geyter, N., Lippens, S., Vanden Bossche, R., Marhavý, P., Kremer, A., Morreel, K., Guérin, C.J., Tava, A., Oleszek, W., Thevelein, J.M., Campos, N., Goormachtig, S., Goossens, A. (2013) The protein quality control system manages plant defence compound synthesis. Nature, 504, 148152

Porter, K.R. \& Anderson, K. (1982) The structure of the cytoplasmic matrix preserved by freeze-drying and freeze substitution. Europ. J. Cell Biol. 29, 83-96.

Puhka, M., Joensuu, M., Vihinen, H., Belevich , I. \& Jokitalo, E. (2012) Progressive sheet-totubule transformation is a general mechanism for endoplasmic reticulum portioning in dividing mammalian cell. Mol. Bio. Cell. 23, 2424-2432.

Roland, J.C. \& Vian, B. (1991) General preparation and staining of thin sections. Chp. 1 in Electron Microscopy of Plant Cells (Eds. C Hawes \& J I Hall) pp 67-84. Academic Press, London.

Sexton R. \& Hall, J.L. (1991) Enzyme cytochemistry. Chp. 4 in Electron Microscopy of Plant Cells (Eds. C Hawes \& J I Hall) pp 67-84. Academic Press, London.

Rizzo, N.W., Duncan, K.E., Bourett, T.M. \& Howard, R.J. (2015) Backscattered electron SEM imaging of resin sections from plant specimens: observation of histological to subcellular structure and CLEM. J Microsc.this volume

Roland, J.C. \& Vian, B. (1991) General preparation and staining of thin sections. Chp.1 in Electron Microscopy of Plant Cells (Eds. C Hawes \& J I Hall) pp 1-66. Academic Press, London

Rouquette, J., Genoud, C., Vazquez-Nin, G.H., Kraus, B., Cremer, T., \& Fakan, S. (2009) Revealing the high-resolution three-dimensional network of chromatin and interchromatin space: a novel electron-microscopic approach to reconstructing nuclear architecture. Chromosome Res. 17, 801-810.

Scheuring, D., Löfke, C., Krüger, F., Kittelmann, M., Eisa, M., Hughes, L., Smith, R.S., Hawes, C., Schumacher, K. \& Kleine-Vehn, J. (2016) Actin-dependent cellular occupancy of the vacuole determines auxin-induced growth repression. PNAS 113, 452-457. 
Schindelin, J., Arganda-Carreras, I., Frise, E., Kaynig, V., Longair, M., Pietzsch, T., Preibisch, S., Rueden, C., Saalfeld, S., Schmid, B. \& Tinevez, J.Y., (2012) Fiji: an open-source platform for biological-image analysis. Nature methods 9, 676-682.

Shigetomi, E., Bushong, E. A., Haustein, M. D., Tong, X., Jackson-Weaver, O., Kracun, S., Xu, J., Sofroniew, M. V., Ellisman, M. H. \& Khakh, B. S. (2013) Imaging calcium microdomains within entire astrocyte territories and endfeet with GCaMPs expressed using adeno-associated viruses. The Journal of general physiology, 141, 633-647.

Shu, X., Lev-Ram, V., Deerinck, T.J., Qi, Y., Ramko, E.B., Davidson, M.W., Jin, Y., Ellisman, M.H. \& Tsien, R.Y. (2011) A genetically encoded tag for correlated light and electron microscopy of intact cells, tissues, and organisms. PLoS Biol. e1001041. doi: 10.1371/journal.pbio.1001041.

Sommer, C., Strähle, C., Köthe, U. \& Hamprecht, F. A. (2011) Ilastik: Interactive Learning and Segmentation Toolkit. Eighth IEEE International Symposium on Biomedical Imaging (ISBI). Proceedings 230-233.

Starborg, T., Kalson, N.S., Lu, Y., Mironov, A., Cootes, T.F., Holmes, D.F. \& Kadler, K.E. (2013) Using transmission electron microscopy and 3View to determine collagen fibril size and three-dimensional organization. Nat Protoc 8, 143314-48.

Stenn, K. \& Bahr, G.F., 1970. Specimen damage caused by the beam of the transmission electron microscope, a correlative reconsideration. J Utrastructure Res, 31, 526-550.

Thierry, R., Kirschmann, M., Hummel, E., Hawes, C. \& Genoud, C. (2014) Demon registration for $3 \mathrm{D}$ images obtained by serial block face scanning electron microscopy. IEEE International Conference on Image Processing (ICIP), 3587-359. DOI: $10.1109 /$ ICIP.2014.7025728.

Tsai, W.T., Hassan, A., Sarkar, P., Correa, J., Metlagel, Z., Jorgens, D.M. \& Auer, M. (2013) From voxels to knowledge: a practical guide to the segmentation of complex electron microscopy 3D-data. JoVE 90, e51673-e51673.

Wacker, I., Chockley, P., Bartels, C., Spomer, W., Hofmann, A., Gengenback, U., Singh, S., Thaler, M., Grabher, C. \& Schröder, R.R. (2015) Array tomography: characterizing FACdorted populations of zebrafish immune cells by their 3-D ultrastructure. J. Microsc. 258, 105-113. 
Wanner, G., Schäfer T. \& Lütz-Meindl, U. (2013) 3-D analysis of dictyosomes and multivesicular bodies in the green alga Micrasterias denticulata by FIB/SEM tomography. J. Struct. Biol. 184, 203-11.

Volkmann, N. (2002) A novel three-dimensional variant of the watershed transform for segmentation of electron density maps. J Structural Biol. 138, 123-129.

Zechmann, B., Zellnig, G. (2009) Microwave-assisted rapid plant sample preparation for transmission electron microscopy. J. Microsc. 233, 258-268.

Zhuravleva, E., Gut, H., Hynx, D., Marcellin, D., Bleck, C. K., Genoud, C., Cron, P., Keusch, J. J., Dummler, B., Esposti, M. D. \& Hemmings, B. A. (2012) Acyl coenzyme A thioesterase Them5/Acot15 is involved in cardiolipin remodeling and fatty liver development. Mol Cell Biol, 32, 2685-2697.

\section{Legends:}

Figure 1. Work flow from specimen fixation through to data output in an SBFSEM experiment.

Figure 2. TEM versus SBFSEM. A. Transmission EM image of a thin section of an osmium impregnated (ZIO) pea root tip cell. Endoplasmic reticulum (ER), nuclear envelope (NE), Golgi bodies (arrows) and the remains of protein storage vacuoles (PSV) are heavily osmium impregnated. B. SBFSEM image of a similar cells as shown in A. Bars $=4 \mu \mathrm{m}$. C. Low magnification SBFSEM image of longitudinally oriented Arabidopsis root tip, same osmium impregnation as $A$ and $B$. Note that absence of grids and post embedding staining allows obstruction-free view and a clean image compared to an equivalent TEM image.

Figure 3. A \& B. High voltage (1meV) electron micrographs of $1 \mu \mathrm{m}$ thick sections of osmium impregnated maize root tip meristem cell endoplasmic reticulum and nuclear envelope (ne) showing transition between tubular and cisternal ER (cer) plus a Golgi stack (g). Bars $=500 \mathrm{~nm}$

C \& D. Maximum intensity projection and segmented images of root tip tubular and cisternal endoplasmic reticulum reconstructed from an SBFSEM image series. Bars $=1 \mu \mathrm{m}(\mathrm{C}), 2 \mu \mathrm{m}$ (D) 
Figure 4. A. Maize root tip meristematic cells en bloc contrasted using the uranyl acetate, copper and lead citrate technique to stain organellar membranes. B. Reconstruction of a small volume of cortical endoplasmic reticulum lining a cell wall in material prepared as in A. Bars $=4 \mu \mathrm{m}(\mathrm{A}), 2 \mu \mathrm{m}(\mathrm{B})$

\section{Figure 5.}

Reconstructions of plant cells, organelles and tissues. A. Reconstruction of an arabidopsis root tip meristematic cell, showing the nucleus (blue), mitochondria (red), Golgi bodies (green) and endoplasmic reticulum (yellow). B. Segmented vacuole in a similar root tip cell as in A. Note that what appear to be multiple vacuoles in thin section (not shown) is in fact one continuous organelle. See also Scheuring et al., (2016). C and D. Section of an osmium impregnated bean leaf chloroplast $(\mathrm{Ch})$ and a reconstruction of the same chloroplast. Note that thylakoid membranes are selectively stained and what seems to be two chloroplasts in the thin sections is in fact one as seen in the reconstruction. E. Axial reconstruction from $>1300$ cross sectional images of an arabidopsis root showing the cell walls of developing vascular tissue (green) and nuclei of the pericycle cells (magenta). F. Cross sectional view of the reconstruction in $\mathrm{E}$ showing the developing vascular tissue surrounded by the pericycle $(P)$. Bars $=2 \mu m(A, B), 1 \mu m(C, D), 20 \mu m(E), 10 \mu m(F)$.

\section{Figure 6.}

A. Pointed and flat topped specimen pins. B. Side view of pin plus specimen (black arrow) mounted in the microtome chuck. C. Face view of trimmed block mounted on a flat faced pin.

\section{Figure 7.}

Effect of changing parameters such as accelerating voltage, chamber pressure and imaging aperture on the quality of the unprocessed backscattered SEM image. Note dramatic changes in signal quality and charging under these slightly different imaging conditions. Microscope magnification $\times 8 \mathrm{~K}$, image dimensions $6 \mathrm{~K} \times 6 \mathrm{~K}$ pixels, $2 \mu$ s dwell time and $4,2 \mathrm{x}$ $4,2 \mathrm{~nm}$ pixel size.

\section{Figure 8.}

Reconstruction of a dataset from a series of block face images of Golgi bodies in an osmium impregnated pea root. A. Volume Rendering from pixels with maximum intensity using 
AMIRA. B. Volume Rendering from pixels with maximum intensity of masked area containing only the Golgi bodies C. Semi-automatic segmentation of Golgi bodies using IMARIS using the same dataset. Maximum intensity projection of the entire dataset is visible in grey. Bar = $1 \mu \mathrm{m}$ 


\section{Table 1}

\begin{tabular}{|c|c|c|c|c|c|c|c|c|c|c|c|c|}
\hline $\begin{array}{l}\frac{\varepsilon}{\pi} \\
\frac{0}{00} \\
\frac{0}{2}\end{array}$ & 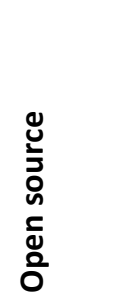 & $\begin{array}{l}\frac{\bar{\pi}}{0} \\
\frac{0}{d} \\
\frac{\varepsilon}{\varepsilon} \\
0\end{array}$ & 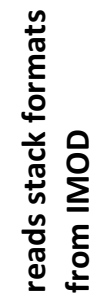 & 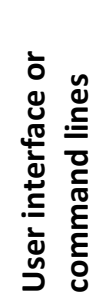 & 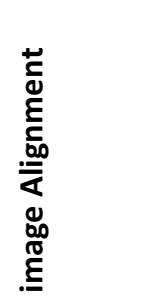 & 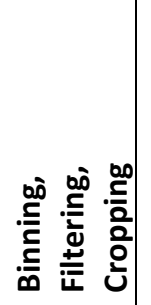 & 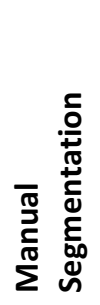 & 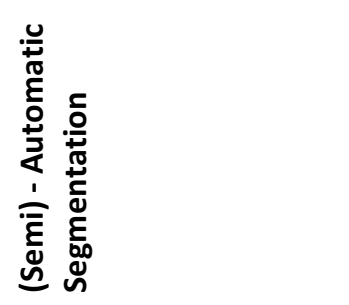 & 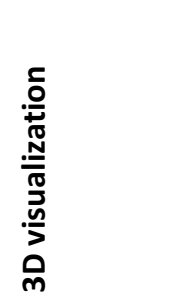 & 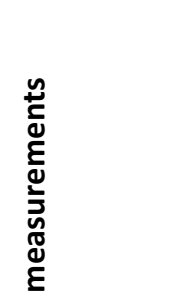 & $\frac{\mathscr{\varrho}}{\partial}$ & $\begin{array}{l}\frac{0}{0} \\
\frac{0}{0} \\
\frac{0}{0} \\
\frac{0}{x} \\
\ddot{x}\end{array}$ \\
\hline $\begin{array}{l}\text { Amira } \\
\text { FEI }\end{array}$ & & $\checkmark$ & $\begin{array}{l}\checkmark \\
. \mathrm{rec} \\
. \mathrm{mrc}\end{array}$ & GUI & $\checkmark$ & $\checkmark$ & $\checkmark$ & $\begin{array}{l}\text { threshold, } \\
\text { magic wand (region } \\
\text { growth), } \\
\text { threshold-based } \\
\text { volume rendering, } \\
\text { filament tracing }\end{array}$ & $\checkmark$ & $\stackrel{\checkmark}{2 D}$ and 3D & $\checkmark$ & $\begin{array}{l}\text { Scripting } \\
\text { within } \\
\text { Extension } \\
\text { XPand in } \\
\text { C++ }\end{array}$ \\
\hline $\begin{array}{l}\text { Imaris } \\
\text { Bitplane }\end{array}$ & & $\checkmark$ & $\begin{array}{l}\checkmark \\
. r e c \\
. \mathrm{mrc} \\
. s t\end{array}$ & GUI & $\begin{array}{l}\text { only with } \\
\text { external } \\
\text { plugins } \\
\text { (needs } \\
\text { XTension } \\
\text { module) }\end{array}$ & $\checkmark$ & $\checkmark$ & $\begin{array}{l}\text { threshold, } \\
\text { magic wand (region } \\
\text { growth), }\end{array}$ & $\checkmark$ & $2 \mathrm{D}$ and $3 \mathrm{D}$ & $\checkmark$ & $\begin{array}{l}, \text { but } \\
\text { requires } \\
\text { Xtension } \\
\text { module }\end{array}$ \\
\hline $\begin{array}{l}\text { Digital } \\
\text { Micrograph } \\
\text { Gatan }\end{array}$ & $\begin{array}{l}\text { Free } \\
\text { offline } \\
\text { version }\end{array}$ & $\checkmark$ & $\begin{array}{l}\checkmark \\
. \mathrm{mrc}\end{array}$ & GUI & $\checkmark$ & $\checkmark$ & $\checkmark$ & $\begin{array}{l}\text { threshold, } \\
\text { magic wand (region } \\
\text { growth), }\end{array}$ & no & $\begin{array}{l}\checkmark \\
\text { only 2D }\end{array}$ & no & scripting \\
\hline $\begin{array}{l}\text { Microscopy } \\
\text { Image } \\
\text { browser } \\
\text { Belevich et } \\
\text { al. } 2016 \\
\end{array}$ & $\checkmark$ & & $\begin{array}{l}. \\
. r e c \\
. m r c \\
. s t\end{array}$ & GUI & $\checkmark$ & $\checkmark$ & $\checkmark$ & $\begin{array}{l}\text { threshold, } \\
\text { membrane click } \\
\text { tracker, magic wand } \\
\text { (region growth), Frangi } \\
\text { tubular filter }\end{array}$ & $\begin{array}{l}\text { Using } \\
\text { other } \\
\text { programs } \\
\text { as Matlab, } \\
\text { Fiji, 3D } \\
\text { viewer or }\end{array}$ & $2 \mathrm{D}$ and $3 \mathrm{D}$ & $\begin{array}{l}\text { No, but interacts } \\
\text { with other progams } \\
\text { as Fiji, 3D viewer } \\
\text { and Imaris }\end{array}$ & $\checkmark$ \\
\hline
\end{tabular}




\begin{tabular}{|c|c|c|c|c|c|c|c|c|c|c|c|}
\hline & & & & & & & & Imaris & & & \\
\hline $\begin{array}{l}\text { IMOD/ } \\
\text { Etomo } \\
\text { Kremer et } \\
\text { al. } 1996\end{array}$ & $\checkmark$ & $\begin{array}{l}. \\
. \mathrm{rec} \\
. \mathrm{mrc} \\
. \mathrm{st}\end{array}$ & both & $\begin{array}{l}\checkmark \\
\text { (etomo) }\end{array}$ & $\checkmark$ & $\checkmark$ & threshold & $\checkmark$ & $2 \mathrm{D}$ and $3 \mathrm{D}$ & $\begin{array}{l}\text { no, only extracts } \\
\text { snapshots of the } \\
\text { movie which have } \\
\text { to be assembled } \\
\text { into a movie using } \\
\text { other programs }\end{array}$ & $\checkmark$ \\
\hline $\begin{array}{l}\text { ImageJ/FIJI } \\
\text { Schindelin } \\
\text { et al. } 2012\end{array}$ & $\checkmark$ & $\begin{array}{l}. \\
. r e c \\
. m r c \\
. s t\end{array}$ & both & $\checkmark$ & $\checkmark$ & $\checkmark$ & $\begin{array}{l}\text { through segmentation } \\
\text { plugins }\end{array}$ & $\begin{array}{l}\checkmark \\
\text { e.g. 3D } \\
\text { viewer }\end{array}$ & $\begin{array}{c}\checkmark D \\
2 \mathrm{and} \\
3 \mathrm{D}\end{array}$ & $\checkmark$ & $\begin{array}{l}\checkmark \\
\text { plugins, } \\
\text { macros }\end{array}$ \\
\hline $\begin{array}{l}\text { Reconstruct } \\
\text { Fiala } 2005\end{array}$ & $\checkmark$ & no & GUI & $\begin{array}{l}\text { manual and } \\
\text { by } \\
\text { correlation } \\
\text { (only } \\
\text { translation) }\end{array}$ & no & $\checkmark$ & local thresholding & $\checkmark$ & $\begin{array}{l}\checkmark \\
\text { only 2D }\end{array}$ & no & no \\
\hline $\begin{array}{l}\text { Ilastik } \\
\text { Sommer et } \\
\text { al. } 2011\end{array}$ & $\checkmark$ & no & both & no & $\checkmark$ & $\checkmark$ & $\begin{array}{l}\text { machine-learning- } \\
\text { based pixel and object } \\
\text { classification, } \\
\text { watershed-based } \\
\text { carving }\end{array}$ & $\checkmark$ & $2 \mathrm{D}$ and $3 \mathrm{D}$ & no & $\checkmark$ \\
\hline
\end{tabular}




\section{Lay abstract:}

Transmission electron microscopy is traditionally used for the study of extremely thin sections of resin embedded biological material. Although permitting very high resolution studies of cell structure and cellular components, to understand the three dimension organisation of cells required technically difficult serial sectioning and image reconstruction. A new technology termed serial block face scanning electron microscopy permits the imaging of block faces of resin embedded material with a high resolution scanning electron microscope. With a microtome built into the microscope specimen chamber, the block face can be repeatedly sectioned and images collected automatically. These serial images can be reconstituted into three dimensional reconstructions of cell for further analyses. Here we report on the application of this technology to plant material for the reconstruction of plant cell membrane systems in three dimensions. 